\title{
Los «regionalismos» gallegos de la lengua española. Cambios introducidos en el DRAE-2001
}

\author{
MERCEDES GONZÁLEZ SARMIENTO \\ Universidade de Vigo
}

\section{INTRODUCCIÓN}

Los lexicógrafos registran en los diccionarios generales de lengua española multitud de palabras que no son originariamente castellanas, pero que se han ido incorporando a los repertorios, ya que el concepto al que hacen referencia es conocido por un amplio número de hablantes y usuarios. Estas palabras son en su mayoría dialectalismos, regionalismos y extranjerismos.

Dentro de este grupo de palabras, habría que incluir aquellas voces gallegas que han pasado a formar parte del acervo del léxico español y que no reciben el tratamiento adecuado en las obras lexicográficas de carácter general, ya que aparecen consignadas como regionalismos del castellano cuando, en realidad, deberían incluirse como integraciones, interferencias o préstamos. Este hecho es una consecuencia directa de la ambigüedad con la que se define el término regionalismo, utilizado no solo para hacer referencia a toda aquella voz que pertenece a un habla regional, sino también a toda aquella voz que se emplea en una determinada región.

En Galicia, el contacto lingüístico entre el gallego y el castellano provoca interferencias entre estas dos lenguas en el nivel fónico, en el morfológico, en el sintáctico y, muy especialmente, en el léxico. En este último se puede observar cómo el gallego ha recibido un gran número de palabras procedentes del castellano, al mismo tiempo que el castellano de Galicia ha recibido, aunque en menor medida, palabras procedentes del gallego.

El $D R A E$, con un reconocido valor normativo que lo hace único en su género, es uno de los diccionarios que posee mayor cantidad de marcas diatópicas para localizar geográficamente las voces de uso restringido de las distintas áreas y países de habla hispánica. 
La constante preocupación de la Real Academia Española por registrar la cambiante realidad de la lengua española queda patente en el prólogo de la última edición del DRAE, en donde se nos advierte de que:

El Diccionario contiene una amplia selección de las voces y acepciones de uso regional o provincial español, así como de aquéllas que corresponden a las distintas áreas y países de habla hispánica, cada una de ellas con su correspondiente marca, generalmente abreviada [...]. Requisito para el mantenimiento de estas entradas en el repertorio es que su empleo actual -se excluyen, por tanto, los arcaísmos dialectalespueda ser testimoniado por la documentación académica o a través de otras vías, especialmente la información proporcionada por los académicos españoles y de los países hispánicos (DRAE-2001: xxxIV).

Tal y como se nos indica en este fragmento entresacado de las «Advertencias para el uso de este diccionario», en el DRAE-2001 se ha llevado a cabo una revisión y actualización de todas las marcas diatópicas, tomando como base documental, entre otras, el Banco de Datos del Español, que el Instituto de Lexicografía viene elaborando desde hace diez años.

Si se compara el DRAE-2001 con el DRAE-1992, se verá que son muchas las modificaciones que se han introducido a este respecto. Todos estos cambios han afectado de manera especial a los «regionalismos» gallegos, que se han visto reducidos en un 28.6\%: el DRAE-1992 registraba 98 acepciones y formas complejas marcadas diatópicamente en 94 voces, mientras que el DRAE-2001 tan solo recoge 70 acepciones y formas complejas en 66 voces.

Comparativa entre el DRAE-1992 y el DRAE-2001

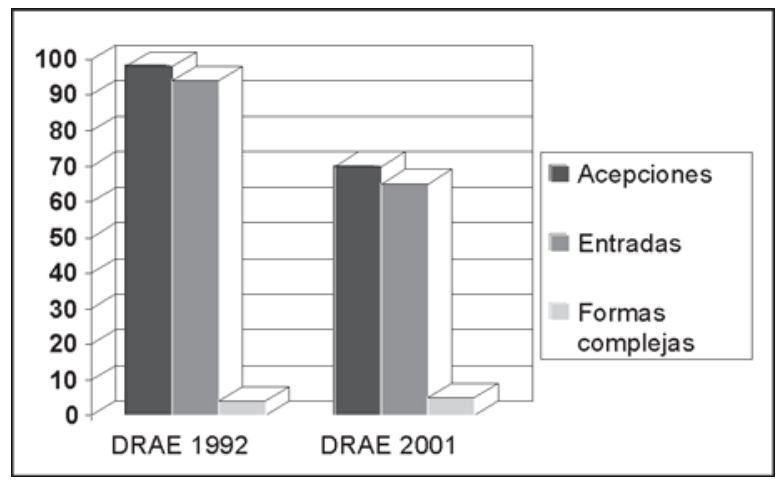

Tal reducción es, a nuestro juicio, bastante significativa y por ello creemos que merece un estudio detenido. 


\section{Cambios introducidos en el DRAE-2001}

Con el fin de ofrecer una visión general de los principales cambios que han experimentado los «regionalismos» gallegos en la última edición del diccionario académico, hemos establecido tres grupos diferentes: el primero, formado por los «regionalismos» gallegos que se mantienen en el DRAE-2001 (46); el segundo, formado por los «regionalismos» gallegos que se han eliminado en el diccionario académico (52); y el tercero, formado por aquellos lemas, acepciones y formas complejas que se han incorporado en la última edición de esta obra lexicográfica por tener un uso restringido en Galicia (24). A continuación, veremos cada uno de estos tres grupos con detalle.

\subsection{Grupo 1: «Regionalismos» gallegos que se mantienen en el DRAE-2001}

El DRAE-2001 ha conservado una buena parte de los «regionalismos» gallegos que se recogían en el diccionario académico de 1992. En algunas ocasiones, se han mantenido sin introducir ningún tipo de modificación como, por ejemplo, en abanear:

abanear. intr. coloq. vulg. Gal. oscilar (\| efectuar movimientos de vaivén) (DRAE2001).

Y en otras, se han llevado a cabo las modificaciones que la Academia ha considerado oportunas en cada caso, principalmente en lo que a la marcación diatópica se refiere. De tal manera, observamos cómo en algunas palabras se ha mantenido únicamente la marca «Gal.» y se ha suprimido el resto de las indicaciones geográficas:

alzadero. m. Ast. y Gal. Vasar o anaquel en cocinas y tiendas (DRAE-1992).

alzadero. m. Gal. Vasar o anaquel en cocinas y tiendas (DRAE-2001).

Esto no quiere decir que las marcas geográficas de otras regiones no aparezcan junto a la marca «Gal.». No obstante, encontramos casos en los que esta marca viene acompañada de otras indicaciones geográficas; muestra de ello es la palabra amañar:

amañar. [...] 2. Cantabria y Gal. Arreglar, componer (DRAE-1992).

amañar. [...] 2. tr. Cantb. y Gal. Arreglar, componer (DRAE-2001).

Todas estas enmiendas son fruto de la constante preocupación de la Real Academia Española por localizar con la mayor precisión posible las distintas voces de uso regional del español.

Pero en el DRAE-2001 se ha llevado a cabo otro tipo de modificaciones que nada tienen que ver con las anteriores: son aquéllas que responden a un intento de 
homogeneizar el diccionario. Una de las principales novedades que presenta el $D R A E$ 2001 es que en éste las marcas diatópicas se insertan inmediatamente después del número de acepción y en letra cursiva. De ahí que las indicaciones geográficas que aparecían como parte de la definición en el $D R A E-1992$ hayan cambiado de lugar en la última edición del diccionario de la Real Academia Española. Esto lo podemos observar si comparamos la entrada $c o c a^{3}$ de ambos diccionarios:

coca $^{3}$. f. En Galicia y otras partes, tarasca que sacan el día del Corpus (DRAE1992).

coca $^{3}$. f. Gal. Tarasca del Corpus (DRAE-2001).

\subsection{Grupo 2: «Regionalismos» gallegos que se eliminan en el DRAE-2001}

Un importante número de «regionalismos» gallegos han desaparecido de la última edición del diccionario académico. Se han eliminado los lemas azaya y pintarrojo, ambos de uso restringido en Galicia:

azaya. f. Gal. cantueso (DRAE-1992).

pintarrojo. m. Gal. Pardillo, pájaro (DRAE-1992).

Pero también se han suprimido algunas acepciones como, por ejemplo, la tercera de la voz aceña:

aceña. [...] 3. Ast. y Gal. Molino instalado en la orilla de una ría, y que muele con el flujo y reflujo del mar (DRAE-1992).

Al mismo tiempo, se ha borrado la marca «Gal.» de muchas acepciones de uso regional y se han conservado otras marcas diatópicas que se incluían al lado de ésta. De tal manera, palabras como abad siguen considerándose regionalismos en el DRAE2001, pero ya no gallegos:

abad. [...] 2. En Galicia, Navarra y otras provincias, cura (DRAE-1992).

abad. [...] 6. m. Ar. cura párroco (DRAE-2001).

Un número reducido de voces que figuraban como «regionalismos» gallegos en el $D R A E-1992$ han pasado a ser tratadas como palabras de uso general. Son pocos los ejemplos, pero tenemos algunos como el de balconada:

balconada. f. Gal. Balcón o miradero que domina un vasto horizonte (DRAE-1992).

balconada. [...] 3. f. Balcón o miradero que domina un vasto horizonte (DRAE2001). 
Por otro lado, no en todas las ocasiones en las que la marca diatópica «Galicia» ${ }^{1}$ aparecía en el $D R A E$-1992 en el interior de la definición ha pasado a colocarse inmediatamente después del número de acepción en el $D R A E-2001$; en algunos casos, la indicación geográfica se ha conservado como parte de la definición. Todas aquellas palabras en las que no se ha alterado el lugar de esta marca han pasado a ser tratadas como voces de uso general, y se han eliminado de la lista de «regionalismos» gallegos. Lemas como abadía, antiguamente regionalismo, es ahora de uso general en la lengua española:

abadía. [...] 4. f. Especie de luctuosa que en algunos puntos, especialmente en Galicia, se paga al párroco a la muerte de un feligrés (DRAE-2001).

\subsection{Grupo 3: «regionalismos» gallegos que se incorporan al DRAE-2001}

Es cierto que se han eliminado muchos de los «regionalismos» gallegos que se recogían en el $D R A E$-1992, pero también se han incluido algunos otros después de comprobar, gracias a los corpus lingüísticos con los que cuenta la Real Academia, el uso restringido que éstos poseen en la Comunidad Autónoma Gallega.

De esta manera, y después de evidenciar su uso en el español de Galicia, se ha podido incluir dentro de la lista de «regionalismos» gallegos la palabra destorgar, que ha dejado de considerarse de uso general:

destorgar. m. Romper o arrancar el torgo (DRAE-1992).

destorgar. tr. Gal. Romper o arrancar el torgo (DRAE-2001).

Esta actualización de las localizaciones geográficas ha llevado, en algunos casos, a la inclusión de la marca «Gal.» en voces como carrizo, que, según el DRAE1992, era utilizada en Asturias:

carrizo. [...] 3. Ast. Pajarillo muy común, de color pardo, que anida en los vallados (DRAE-1992).

carrizo. [...] 3. m. Ast. y Gal. chochín (DRAE-2001).

En otros casos, tal actualización ha favorecido la incorporación de alguna nueva acepción en lemas a los que los gallegohablantes les atribuían un significado especial que no se correspondía con los recogidos en el $D R A E-1992$. Esto es lo que ha ocurrido con cativo ${ }^{l}$ y parrillada:

1 El DRAE-1992 empleaba la marca «Galicia» para hacer referencia a Galicia, pero también la marca «gallegos», aunque esta última no apareciera especificada. 
cativo $^{1}$. adj. Gal. Dicho de una persona: Que no tiene cualidades físicas, intelectuales o morales dignas de aprecio (DRAE-2001).

parrillada. [...] 3. f. Gal. y Am. parrilla (\| restaurante) (DRAE-2001).

Por otra parte, hay que tener en cuenta que la incorporación de una nueva acepción de uso regional se puede deber también al desdoblamiento de una acepción, tal y como sucede en torgo:

torgo. m. Extr. y Gal. Tocón, cepa o raíz gruesa, o parte abultada de las ramas (DRAE-1992).

torgo. m. Gal. Tocón, cepa o raíz gruesa. \| 2. m. Gal. Parte abultada de las ramas (DRAE-2001).

Pero no solo se han incorporado nuevas acepciones, sino también la voz carriza, empleada en Galicia y León, y las formas complejas para el año y meter una cuña, utilizadas únicamente en la Comunidad Gallega:

carriza. f. Gal. y León. chochín (DRAE-2001).

año ${ }^{1}$. para el . loc. adv. Gal. y Ur. El año que viene (DRAE-2001).

cuña. meter una $\sim$. fr. Gal. Hacer recomendaciones a favor de alguien (DRAE-2001).

A modo de resumen, ofrecemos un cuadro en el que se detalla, entre paréntesis, el número de acepciones de uso regional en Galicia incluidas dentro de cada uno de los grupos que hemos distinguido.

«Regionalismos» gallegos en el DRAE-2001

\{

Grupo 1:

Sin cambios (18)

Se mantienen (46) Con cambios (28)

Se conserva la marca «Gal.» (21)

La marca «Galicia» cambia de lugar (7)

Lemas (2)

Acepciones (8)

Grupo 2:
Se eliminan (52)

Se eliminan (24)

La marca «Gal.» (14)

Se conserva la marca «Galicia» en el interior de la definición (28)

Pasan a considerarse Palabras de uso general (2)

«regionalismos» Palabras de uso regional (15)

Grupo 3:

gallegos (23)

Palabras que no se incluían (6)

Se incorporan (24)

Se desdobla una definición de uso regional en Galicia (1) 


\section{Dudas sobre el uso Regional de algunas voces en Galicia}

Al repasar la lista de «regionalismos» gallegos que se incluyen en el $D R A E$ 2001, nos han surgido ciertas dudas sobre si determinadas voces debían o no figurar en ésta. De ahí que, con el fin de comprobar su uso restringido en Galicia, hayamos cotejado toda la información ofrecida por el diccionario académico con la recogida en otras obras lexicográficas.

Los diccionarios que hemos empleado para llevar a cabo este estudio contrastivo son los siguientes:

- El DRAE-1992, que nos ha permitido observar los cambios introducidos en la última edición del diccionario académico gracias a la utilización de los corpus lingüísticos que, desde hace algunos años, viene confeccionando el Instituto de Lexicografía de la Real Academia Española.

- El DUE-1998, uno de los diccionarios con mayor prestigio para aquéllos que se dedican a la labor lexicográfica, que nos ha aproximado al tratamiento que reciben los «regionalismos» gallegos en la lexicografía española.

- El DRLE, ${ }^{2}$ que nos ha proporcionado información concreta sobre los «regionalismos» gallegos utilizados en la lengua española. Este diccionario lo hemos empleado para comprobar el uso restringido de las acepciones precedidas de la marca diatópica «Gal.» en el DRAE-2001.

- Por último, el $D R A G$, que nos ha servido de gran utilidad para verificar si existen o no en la lengua gallega las palabras que se registran como «regionalismos» gallegos en el $D R A E-2001$, y en el caso de existir, si el significado que recoge de éstos se corresponde con el ofrecido por la Real Academia Gallega.

Una vez reunida y cotejada toda esta información, observamos cómo el uso restringido de estas voces no aparece avalado por las obras lexicográficas consultadas. A continuación, y a modo de ejemplo, analizaremos con detalle el término parrillada en estas cuatro obras lexicográficas:

parrillada. [...] 3. f. Gal. y Am. parrilla (\| restaurante) (DRAE-2001).

2 Esta obra dispone de una versión electrónica que se puede consultar parcialmente a través de la red, ya que no se encuentra disponible la versión completa. Por eso, si se quieren obtener todas las voces que son utilizadas en Galicia con un determinado significado es necesario realizar una búsqueda manual. 
En la edición del año 1992 no se encuentra este significado de parrillada.

parrillada. f. Plato compuesto de diversos pescados o mariscos, asados a la parri1la. || 2. Plato compuesto de carne de vaca, chorizo, morcilla, etc., asados a la parrilla (DRAE-1992).

Por su parte, María Moliner no registra esta palabra en la lista de entradas de su diccionario.

Los autores del DRLE no consideran que este sustantivo tenga un uso regional $\mathrm{y}$, por ello, no lo incluyen.

Tampoco en el DRAG figura este vocablo como entrada.

Tal y como acabamos de ver, ninguno de los diccionarios consultados hace referencia al carácter regional de esta voz en Galicia, a excepción del DRAE-2001. En el prólogo de éste se puede leer que, para la incorporación y eliminación de regionalismos, se ha tenido en cuenta el Corpus de Referencia del Español Actual (CREA). Lo más apropiado nos ha parecido, pues, buscar parrillada en este corpus que es utilizado como fuente por la Real Academia Española. Los criterios que hemos tenido en cuenta para la consulta de este 'regionalismo' gallego en el CREA han sido los siguientes:

- El cronológico: hemos seleccionado el período que va desde 1992 a 2001, ya que son los años que distan de una edición a otra.

- El medio: hemos marcado todos, ya que éste no es un parámetro importante para nuestra búsqueda.

- El geográfico: como cabe esperar, en este apartado hemos seleccionado España (no existe la posibilidad de limitar la búsqueda a una determinada región o comunidad autónoma española), puesto que las palabras que estudiamos son voces de uso restringido solo en la Península Ibérica.

- El tema: al igual que el tema, este criterio no es determinante para la consulta que hemos realizado.

De esta voz únicamente hemos conseguido 3 casos en 3 documentos, pero ninguno de ellos con el significado de «restaurante»:

En estas zonas el plato se llama justamente «parrillada» y suele ser uno de los platos preferidos por la invasión turística de cada verano (Xavier Domingo, El sabor de Espa$\tilde{n} a, 1992)$.

El semidesierto queda para los héroes, el resto es playa, parrillada, deporte, jardín, cerveza (Manuel Leguineche, La tierra de Oz. Australia vista desde Darwin hasta Sydney, 2000). 
Sin embargo, faltaban los olores verdaderos del barrio del abuelo: el de las almendras garrapiñadas de la churrería, que se extendía, espeso como una mancha visible, por los pisos altos; el de la parrillada de los domingos del restaurante más próximo (Laura EsPido Freire, Melocotones helados, 1999).

Los únicos ejemplos en los que la palabra parrillada es utilizada como sinónimo de 'restaurante' pertenecen al español de América, concretamente a Argentina, Cuba y Ecuador:

El recreo se irá transformando, a lo largo de la acción, en un bar alemán, una parrilla$d a$, un depósito de chatarra y un bar americano, sin perder nunca su característica construcción provisoria y deprimente (Roberto CossA, Los compadritos, 1985).

Se trata de una villa con 16 habitaciones, una suite y tres cabañas, con cine, piscina, bolera, gimnasio, canchas de tenis, sala de recreación, restaurante, parrillada y tienda (Granma Internacional, 07/1996, núm. 6, Cuba).

En Av. Las Aguas, junto a la parrillada La Vaca Gaucha (Expreso de Guayaquil, 13/ 03/2003, Ecuador)

La utilización del CREA nos ha permitido comprobar cómo la adición, supresión o enmienda en el diccionario académico de las marcas diatópicas no se hace tomando únicamente como punto de referencia este corpus lingüístico. En la página web de la Real Academia Española se explica que la actualización del $D R A E$ se lleva a cabo teniendo en cuenta las propuestas que «parten, en su mayoría, de las Comisiones académicas» aunque, «en ciertos casos, son los propios académicos quienes presentan directamente sus propuestas al Pleno».

\section{LoS «REGIONALISMOS» GALLEGOS EN LA PRÓXIMA EDICIÓN DEL DRAE}

En la página web de la Real Academia Española se muestran algunas de las enmiendas que se incluirán en la próxima edición del $D R A E$. El sistema de consulta de enmiendas y adiciones emplea la técnica del hipertexto para mostrar las modificaciones que se van produciendo en el diccionario.

Después de buscar en la red cada uno de los «regionalismos» gallegos que se recogían en el $D R A E-2001$, vemos que ninguna de las enmiendas previstas para el diccionario académico afecta a alguno de éstos. Las únicas voces de uso regional en Galicia que se han modificado son castaña, lambión y salsero. A continuación, comentamos brevemente cuáles son los cambios introducidos: 
- Bajo el lema castaña se ha incluido una nueva forma compleja que no aparecía en el DRAE 2001: castaña de Indias o castaña loca, empleada en España para hacer referencia al «fruto no comestible del castaño de Indias, semejante a la castaña común».

- En lambión, na se ha añadido la marca diatópica «Ast.», forma abreviada de «Asturias».

lambión, na. adj. Cantb., Gal., León, Pal. y Rioja. goloso (DRAE-2001).

Artículo enmendado. Avance de la vigésima tercera edición

lambión, na. adj. Ast., Cantb., Gal., León, Pal. y Rioja. goloso.

- Y, por último, en salsero, ra se han añadido tres nuevas acepciones. Esto ha provocado un desplazamiento del significado empleado en Galicia: en el $D R A E$-2001 se recogía en la quinta acepción, y en la próxima edición se hará en la segunda.

salsero, ra. adj. coloq. Mur. y Nav. Entremetido. || 2. m. Gal. Salpicadura del agua del mar, especialmente cuando rompen las olas o hay temporal. $\| \mathbf{3}$. f. Vasija en que se sirve la salsa. \|4. f. Taza pequeña para mezclar colores. V. tomillo salsero (DRAE-2001).

\section{Artículo enmendado. Avance de la vigésima tercera edición}

salsero, ra. 1. adj. Perteneciente o relativo a la música de salsa o a su baile. 2. adj. Que compone o interpreta música de salsa. U. m. c. s. 3. adj. Aficionado a la música de salsa o a su baile. U. t. c. s. 4. adj. coloq. Mur. y Nav. Entremetido. 5. m. Gal. Salpicadura del agua del mar, especialmente cuando rompen las olas o hay temporal. V. tomillo salsero.

\section{Conclusiones}

1. En la última edición del diccionario de la Real Academia Española se ha reducido en un $28.6 \%$ el número de los «regionalismos» gallegos: mientras que en el $D R A E-1992$ se recogían 98 acepciones de uso restringido en Galicia, en el DRAE2001 tan solo se recogen 70 acepciones. De tal manera, se han mantenido 46 acepciones al mismo tiempo que se han suprimido $52 \mathrm{y}$ se han incorporado 24 .

2. Después de revisar y cotejar la información ofrecida por el $D R A E-2001$ con la de otros diccionarios como el $D R A E-1992$, el $D U E-1998$, el $D R L E$ y el $D R A G$, y consultar el CREA seguimos teniendo dudas de si algunos vocablos, como parrillada, incluidos como «regionalismos» gallegos en la última edición del dicciona- 
rio de la Real Academia Española, deben o no considerarse de uso restringido en Galicia.

La utilización del Corpus de Referencia del Español Actual para el estudio de estas voces nos ha permitido comprobar cómo en muchas ocasiones la Real Academia Española no tiene en cuenta, para la inclusión o la supresión de voces, los corpus lingüísticos que le ha encargado confeccionar al Instituto de Lexicografía.

3. De acuerdo con las enmiendas al diccionario académico que se muestran en la página web de la Academia, no parece que los «regionalismos» gallegos vayan a sufrir demasiadas modificaciones en la vigésima tercera edición o al menos no parece que esté prevista la eliminación de alguno de ellos. Sin embargo, no estaría de más que en la próxima edición se incorporaran algunas voces gallegas que son de uso general en Galicia como, por ejemplo, los adjetivos enxebre y reseso.

\section{REFERENCIAS}

Aвad NeBot, Francisco (1997): Cuestiones de lexicología y lexicografía, Madrid, UNED.

Ahumada Lara, Ignacio (1989): Aspectos de lexicografia teórica, Granada, Universidad de Granada.

— (2000): «Nuevos horizontes de la lexicografía regional», en Stefan Ruhstaller y Josefina Prado

Aragonés, eds., Tendencias en la investigación lexicográfica del español, Huelva, Universidad de Huelva, pp. 15-35.

Alvar LóPEz, Manuel (1973-1974): «Galicia en la cartografía lingüística», Verba, 1, pp. 54-62.

— (1982): «Atlas lingüísticos y diccionarios», Lingüística española actual, rv, pp. 253-323.

Alvar Ezquerra, Manuel (1993): Lexicografia descriptiva, Barcelona, Biblograf.

— (1996-1997): «Lexicografía dialectal», Estudios de Lingüística, 11, pp. 79-109.

CASARES, Julio (1992): Introducción a la lexicografia moderna, Madrid, CSIC.

Cotarelo, Armando (1927): «El castellano en Galicia (Notas y observaciones)», Boletín de la Real Academia Española, 14, pp. 82-136.

Fernández Sevilla, Julio (1974): Problemas de la lexicografía actual, Bogotá, Instituto Caro y Cuervo.

García GonzÁlez, Constantino (1976): «Interferencias léxicas entre gallego y castellano», Revista española de Lingüística, 6/2, pp. 327-343. 
Haensch, G., L. Wolf, S. Ettinger y R. Werner (1982): La lexicografía. De la lingüística teórica a la lexicografía práctica, Madrid, Gredos.

Lara Ramos, Luis Fernando (16/07/2003): «Los diccionarios contemporáneos del español y la normatividad», en http://cvc.cervantes.es/obref/congresos/valladolid/ponencias/ unidad_diversidad_del_espanol/2_el_espanol_de_america/lara_l.htm.

Martínez de Sousa, José (29/04/2003): «Novedades en el Diccionario de la Academia (edición 2001)», en http://www.angelfire.com/ma/apuntes/drae.htm.

Nola CAmpos, $M^{a}$ do Camiño (1982): «Interferencias léxicas entre as linguas galega e castelá», Grial, 75, pp. 91-99.

Porto Dapena, José-Álvaro (2002): Manual de técnica lexicográfica, Madrid, Arco/Libros.

- (11/2/2004): «El español en contacto con el gallego», en http://cvc.cervantes.es/obref/congresos/valladolid/ponencias/unidad_diversidad_del_espanol/4_el_espanol_en_contacto/ porto_a.htm

SALVADOR, Gregorio (1980): «Lexicografía y Geografía Lingüística», Revista española de Lingüística, 10/1, pp. 49-57.

- (2003): «Los Dialectalismos en los Diccionarios», en M Antonia Martín Zorraquino y José Luis Aliaga Jiménez, eds., La lexicografía hispánica ante el siglo XXI. Balance y perspectivas, Zaragoza, Gobierno de Aragón-Institución Fernando el Católico, pp. 209-220.

\section{DiCCIONARIOS}

Castro Macía, Luis (2001): Diccionario Xerais galego-castelán, castellano-gallego, Vigo, Edicións Xerais de Galicia.

DRAE-1992. Real Academia Española (1992): Diccionario de la lengua española, Madrid, EspasaCalpe.

DRAE-2001. Real Academia Española (2001): Diccionario de la lengua española, Madrid, EspasaCalpe.

DRAG. Real Academia Galega (1997): Diccionario da Real Academia Galega, dirigido por Constantino García y Manuel González González, A Coruña-Vigo, Real Academia GalegaEdicións Xerais de Galicia-Editorial Galaxia.

DRLE. Pablo Grosschmid y Cristina Echegoyen (1998): Diccionario de regionalismos de la lengua española, Barcelona, Juventud.

DUE-1998. María Moliner (1998²): Diccionario de uso del español, Madrid, Gredos. 